TRABAJOS ORIGINALES

Rev Obstet Ginecol Venez. 2021; 81 (1): 22-32

https://doi.org/10.51288/00810106

\title{
Vulvovaginitis candidiásica: manifestaciones clínicas según la especie de cándida*
}

\author{
Drs. Alexander Bahsas Zamora, ${ }^{1}$ Gabriel Narváez Narváez, ${ }^{1}$ Ramón Fernández Ramírez. ${ }^{2}$
}

\begin{abstract}
RESUMEN
Objetivo: Caracterizar las manifestaciones clínicas de la vulvovaginitis candidiásica según la especie identificada en mujeres que acudieron al Servicio de Ginecología de la Maternidad Concepción Palacios.

Métodos: Estudio prospectivo, transversal, correlacional no causal, que incluyó una muestra probabilistica de 203 mujeres a las cuales se les tomó una muestra de la secreción vaginal para cultivo, con la finalidad de identificar las diferentes especies de hongos presentes y comparar las manifestaciones clínicas en cada caso.

Resultados: Hubo 165 casos con resultado del cultivo positivo, entre ellas, las dos especies con mayor frecuencia fueron Candida albicans, en 105 pacientes (63,6\%) y Candida glabrata, en 49 pacientes (29,7 \%). Con menor frecuencia se encontraron Candida parapsilosis, Candida tropicalis y Candida krusei. En la correlación entre las manifestaciones clínicas y la especie, la única característica que se asoció en forma significativa fue la presencia de excoriaciones con Candida tropicalis. $(p=0,012)$.

Conclusiones: En general, las características clínicas de la vulvovaginitis candidiásica no se correlacionan con la especie identificada.
\end{abstract}

Palabras clave: Vulvovaginitis candidiásica, Clínica, Especie de cándida.

\section{SUMMARY}

Objective: To characterize the Vulvovaginal Candidiasis clinical manifestations according to the identified Candida species in women who attended to the gynecological service of the Maternidad Concepción Palacios.

Methods: Prospective, cross-cutting, non-causal correlational study, which included a probabilistic sample of 203 women. A sample of vaginal secretion for cultivation was taken from each woman, in order to found the different species of fungi and to compare the clinical manifestations between the different cases.

Results: There were 165 positive cultivation cases. Among them the two species with the highest frequency were Candida albicans in 105 patients (63.64\%) and Candida glabrata in 49 patients (29.70\%). Candida parapsylasis, Candida tropicalis, and Candida krusei were found less frequently. With regard, the correlation between clinical manifestations and the species, the only clinical manifestation that presented statistically significant clinical differences were excoriations in candida tropicalis cases ( $p-0.012)$.

Conclusions: There were no correlation between the vulvovaginal candidiasis clinical manifestations and the identified Candida species.

Keywords: Vaginal candidiasis, Clinical manifestations, Candida species.

\section{INTRODUCCIÓN}

${ }^{1}$ Especialistas en Obstetricia y Ginecología, egresados del Programa de la Universidad Central de Venezuela, con sede en la Maternidad Concepción Palacios. ${ }^{2}$ Especialista en Obstetricia y Ginecología, Adjunto al Servicio de Ginecología, Maternidad Concepción Palacios.

*Trabajo Especial de Grado presentado y aprobado para optar al título de Especialista en Obstetricia y Ginecología.
La vulvovaginitis por cándida es una entidad muy frecuente y sus características clínicas no han sido ampliamente descritas para cada especie en particular, por lo que la aproximación diagnóstica de la especie involucrada, de acuerdo a los signos y síntomas 


\section{VULVOVAGINITIS CANDIDIÁSICA: MANIFESTACIONES CLÍNICAS \\ SEGÚN LA ESPECIE DE CÁNDIDA}

que presenta la paciente, no han sido descritos en la actualidad. La vulvovaginitis candidiásica es uno de los principales motivos de consulta ginecológica tanto a nivel nacional como internacional, siendo la segunda causa de leucorrea, solo detrás de la vaginosis. La mayoría de las mujeres experimentará al menos un episodio de vulvovaginitis candidiásica (VVC) en su vida $(1-3)$.

En las últimas décadas, se ha incrementado en forma significativa la prevalencia de las infecciones por cándida, en parte como consecuencia del avance de la medicina que ha logrado mayor éxito en el tratamiento de enfermedades neoplásicas y otros padecimientos que antes eran mortales. Adicionalmente, el uso terapéutico o diagnóstico de procedimientos invasores, la prescripción extendida de antibióticos de amplio espectro, la alimentación parenteral, el aumento en la esperanza de vida y la aparición del virus de la inmunodeficiencia humana (VIH) son situaciones que favorecen la aparición de infecciones oportunistas por cándidas, considerada uno de los agentes patógenos más frecuentes. Aproximadamente, $75 \%$ de las mujeres tendrán, al menos, un episodio en su vida, $50 \%$ más de un episodio y $5 \%$ tendrán episodios recurrentes $(4-6)$.

Para cualquier médico involucrado en el cuidado de la salud de la mujer, la vulvovaginitis es una queja comúnmente encontrada y uno de los motivos de consulta más frecuentes en ginecología. De las muchas causas de las infecciones vaginales, específicamente la VVC, es una de las más comunes y podría tener un impacto significativo en los costos de salud. Las especies de cándida involucradas en la vulvovaginitis y sus manifestaciones clínicas han sido ampliamente investigadas a nivel mundial, descritos sus tratamientos específicos y también la resistencia antimicótica, por lo que este es uno de los gérmenes de mayor importancia y frecuencia en la producción de esta enfermedad. Algunas especies Candida no albicans parecen tener la particularidad de presentar resistencia a tratamientos con los antimicóticos usuales, lo que hace que, en algunos casos, el tratamiento de inicio no sea efectivo para erradicar la infección y eliminar la sintomatología, por lo que sería de utilidad determinar la presentación clínica según la especie para orientar el tratamiento adecuado. En Venezuela, aun teniendo una elevada incidencia de esta patología y ser uno de los motivos de consulta más frecuentes en el área ginecológica, no se han publicado estudios que documenten la asociación entre la presentación clínica y la especie de cándida involucrada. La descripción de la presentación clínica de la VVC según la especie de cándida identificada en el cultivo de secreción vaginal, aportaría datos a este respecto, permitiendo al clínico una mejor orientación diagnóstica, sin necesidad de utilizar un método adicional, como el cultivo de secreción vaginal, que acrecentaría los costos operativos en el manejo de la patología. Además, la información adecuada sobre esta asociación entre clínica y especie, permitiría obtener mejores respuestas al tratamiento y disminuir el índice de recurrencia, limitando la necesidad del estudio micológico solo para aquellos casos en los que la respuesta a la terapéutica no sea satisfactoria.

La frecuencia de detección reportada de las diferentes especies de cándidas es variable según las características de las pacientes, la presencia o no de síntomas y según el método de detección. En general, se han encontrados cultivos positivos para hongos en frecuencias variables: tan bajas como $12,2 \%$ (7), cifras intermedias como $61,5 \%$ (8) o hasta 79,3\% (9). En pacientes sintomáticas, se obtienen cultivos positivos en $64,6 \%$ y en pacientes asintomáticas en $11,2 \%$ de ellas (10).

La mayor parte de las infecciones fúngicas son producidas por la especie Candida albicans (más del $50 \%$ ). Esta se considera flora habitual del organismo humano; puede ser aislada en el tracto genital de hasta en $20 \%$ de mujeres asintomáticas sanas en edad reproductiva y tener diversas localizaciones, pero se aíslan con mayor frecuencia de la boca, el conducto 
gastrointestinal y la vagina. $(3,11-13)$. Entre los cultivos positivos para cándidas, se aísla $C$. albicans en frecuencia variables entre 43,5\% y hasta 80,2\% (7-9, 14 - 18). La Candida albicans se considera un germen saprofito de diversas mucosas y de la piel; pero pueden ser patógenos cuando las condiciones son propicias y actuan como germen oportunista. Entre los factores del huésped que pueden predisponer a la infección se incluyen factores fisiológicos como el embarazo, y otros como enfermedades o procesos debilitantes, estados de inmunosupresión, uso de anticonceptivos orales o estrógenos, diabetes mellitus pobremente controlada, uso sistémico de glucocorticoides, uso sistémico de antibióticos de amplio espectro y alta frecuencia del coito. Por otro lado, algunos atributos del germen que pueden contribuir a su virulencia son: la adhesión, la formación de hifas, el fenotipo intercambiable, la producción de enzimas hidrolíticas extracelulares y la formación de biopelículas. $(3,5,13$, $19-21)$.

Las especies no albicans de mayor importancia son: Candida tropicalis, Candida glabrata, Candida parapsilosis y Candida krusei cuya frecuencia se ha incrementado en los últimos años. En menor proporción: Candida lusitaniae, Candida guilliermondii, Candida kefyr, Candida famata, Candida zeylanoides y Candida dubliniensi. (11, 12, 22). Se aíslan entre 19,8 \% y $32 \%$ de los casos $(10,14,16,18)$. Entre ellas, las más frecuentes son C. glabrata, entre $13,2 \%$ y $27 \%(7,9$, 15, 16, 23); C. tropicalis: 0,8 \% - 13,5\% (7, 9, 15, 23); C. parapsilosis, $2 \%-8,9 \%(7,15,16)$ у C. krusei entre $0,8 \%$ y $5 \%(9,15)$.

Cuando la detección se hace por reacción en cadena de polimerasa (PCR), se encuentra ADN de cándidas en $33,1 \%$. De estas el 80,2 \% - $89 \%$ corresponden $C$. albicans; 7,9\% - 14,3\% a C. glabrata; $1,7 \%-5,9 \%$ C. parapsilosis y $1,4 \%-8 \%$ C. tropicalis $(24,25)$.
En Venezuela, De La Parte y cols. (1), en pacientes con sintomatología de vulvovaginitis, encontraron que el germen más frecuentemente aislado fue la C. albicans en un $84 \%$ de los casos, las especies no albicans representaron el $16 \%$, de las cuales $8 \%$ correspondió a C. tropicalis, C. guilliermondii con $5 \%$ y $C$. glabrata con $3 \%$. En presencia de flujo, se identifica Candida albicans en 9,8\% de los casos como segundo germen más frecuente, solo por detrás de la vaginosis bacteriana con $22 \%$ (26). En gestantes con sospecha clínica de candidiasis vaginal se encontró un $38 \%$ de frecuencia de infección vaginal por cándida, de las cuales $88 \%$ correspondieron a C. albicans, $8 \%$ a C. glabrata y $4 \%$ a C. krusei (27).

Las manifestaciones clínicas de candidiasis son múltiples, pero las más frecuentes son las cutáneas, mucosas, mucocutáneas y, excepcionalmente, las profundas o sistémicas. La candidiasis vulvovaginal pertenece a las formas mucocutáneas y ocurre cuando hay crecimiento desmedido de cándida, que se produce al haber un desequilibrio en el microambiente vaginal (cambios de acidez normal de la vagina o desequilibrio hormonal). Tales factores son suficientes para que las levaduras se multipliquen y, al generar la infección, las levaduras cambian su micromorfología estructural a blastoconidias (gemaciones) en conjunto con las pseudohifas, cuyas estructuras se consideran virulentas. Este cambio morfológico ocurre en todas las especies, con excepción de C. glabrata, en el que solo aumenta la cantidad de blastoconidias, lo que hace más difícil detectar la infección. (3, 11 - 13, 28).

La presentación clínica de la VVC es variable y puede ir desde completamente asintomática hasta síntomas graves. El prurito vulvovaginal es el síntoma más frecuente de la VVC y, aunque el flujo vaginal blanco espeso y homogéneo, tipo requesón, puede estar presente, el flujo también puede ser fino, acuoso o incluso estar ausente. Adicionalmente 


\section{VULVOVAGINITIS CANDIDIÁSICA: MANIFESTACIONES CLÍNICAS \\ SEGÚN LA ESPECIE DE CÁNDIDA}

puede presentarse disuria externa, irritación vaginal, dolor vaginal, dispareunia, eritema vulvar, fisuras y lesiones satélites. Estos síntomas frecuentemente se exacerban la semana anterior a la menstruación. Cuando se presenta dolor vaginal, aunque no es muy frecuente, debe evaluarse sus características, entre las que se encuentra la intensidad que frecuentemente se documenta utilizando una escala visual análoga $(2,3$, 29, 30).

El Centro de Control de Enfermedades (CDC) de EE.UU. clasifica la VVC en no complicadas y complicadas para facilitar su identificación y manejo. Las VVC no complicadas están caracterizadas por ser de presentación esporádica o infrecuente, causadas por $C$. albicans, presentarse en mujeres inmunocompetentes y manifestar signos y/o síntomas leves o moderados (30). Por otro lado, las complicadas se caracterizan por presentarse en mujeres inmunocomprometidas o diabéticas mal controladas, causadas por especies no albicans, y ser graves o recurrentes. La VVC grave es la que produce eritema vulvar extenso, edema, excoriaciones y fisuras y la VVC recurrente se define como la presentación de 4 o más episodios sintomáticos en 1 año (30).

Clínicamente, los signos y/o síntomas de vulvovaginitis según la especie de cándida identificada parecen ser indistinguibles y no se ha logrado identificar signos o síntomas patognomónicos, por lo que un diagnóstico basado exclusivamente en el examen clínico puede ser causa de errores diagnósticos. Adicionalmente las infecciones por especies no albicans parecen presentar mayor resistencia a los antimicóticos de uso común y estar relacionadas con VVC recurrente $(21,31)$.

Con relación a las manifestaciones clínicas, en general, el prurito es el síntoma más común $(85,9 \%)$, seguido de flujo vaginal $(66,1 \%)$, dolor $(31,1 \%)$ y la dispareunia $(5,0 \%)$. El dolor, la dispareunia y las recurrencias se han relacionado significativamente con especies no albicans (14), también el reporte de tratamiento previo (9), el diagnóstico de VVC grave (18) y la ausencia de síntomas: $61,5 \%$ en mujeres asintomáticas, $38,7 \%$ en mujeres sintomáticas y $11 \%$ en mujeres con VVC recurrente (17). Por otro lado, la edad reproductiva, el embarazo, la diabetes, la anticoncepción, así como el uso de antibióticos se han correlacionado positivamente con la identificación microbiológica de tanto $C$. albicans como $C$. no albicans (14). Algunos autores han reportado que la presentación clínica de las infecciones causadas por C. albicans son indistinguibles de las causadas por especies no albicans (16).

Tradicionalmente el diagnóstico de vulvovaginitis candidiásica se ha fundamentado en los signos y síntomas que se presentan en la paciente, sin confirmación del laboratorio. Otras herramientas diagnósticas incluyen el examen al fresco de la secreción vaginal (añadiendo $\mathrm{KOH}$ al $10 \%$ \%) mediante la tinción de Gram, con la finalidad de identificar gemaciones, hifas o pseudohifas; aunque puede tener un valor cuestionable, ya que pueden ser parte de la flora comensal en alrededor del $20 \%$ de las mujeres, su identificación en conjunto con leucocitos polimorfonucleares es un signo sugestivo de infección. También se usa el cultivo específico para hongos, o pruebas de identificación de ADN de especies de hongos, como la reacción en cadena de polimerasa $(30,32)$. Con respecto a los medios de cultivo para hongos parece no haber diferencia entre los diversos medios disponibles en la actualidad: agar Sabouraud, medio de Nickerson o el medio de cándida Microstix. Sin embargo, un cultivo positivo para hongos no necesariamente indica que el germen identificado es el responsable de los síntomas ya que $15 \%$ - 20\% de las pacientes asintomáticas pueden estar colonizadas y por lo tanto tener cultivos positivos. Debido a esto, es de vital importancia correlacionar los resultados microbiológicos y del cultivo, con los hallazgos clínicos (21). 
La presente investigación se realizó en un intento por caracterizar las manifestaciones clínicas de la vulvovaginitis candidiásica según la especie identificada.

\section{MÉTODOS}

Se realizó un estudio prospectivo, transversal, correlacional no causal. Entre una población de más de 9000 pacientes que suelen asistir al Servicio de Ginecología de la "Maternidad Concepción Palacios" en un año, se seleccionó una muestra probabilística de 203 mujeres, entre 18 y 80 años, con criterio clínico diagnóstico de vulvovaginitis candidiásica. Se excluyeron aquellas con inmunosupresión (infección por virus de inmunodeficiencia adquirida, diabetes mellitus, lupus eritematoso sistémico, en tratamiento con corticoesteroides, etc.), pacientes que habían recibido tratamiento antimicótico para vulvovaginitis por cándida en los tres meses anteriores o tratamiento con antibióticos en los 15 días previos a su inclusión y las que referían coito en las 72 horas previas. Todas las pacientes firmaron un formulario de consentimiento informado.

Una vez incluidas, se corroboraron los criterios clínicos de VVC y, previa colocación del espéculo vaginal, se procedió a tomar muestra de secreción vaginal mediante hisopo estéril, la cual fue trasladada en medio de transporte agar Stuart a un laboratorio privado para su procesamiento. Los medios de cultivo empleados fueron agar Sabouraud y agar Biggy. Los cultivos se procesaron en laboratorio privado ya que el laboratorio de la Maternidad Concepción Palacios no contaba con los medios ni recursos humanos para la realización de los mismos.

Para la identificación de las levaduras se procedió de la siguiente manera: inicialmente se procedió a la producción de tubo germinal o filamentización en suero. Este ensayo consiste en colocar una pequeña porción de una colonia obtenida de un cultivo de 24 horas en $0,5 \mathrm{ml}$ de suero e incubar a $37^{\circ} \mathrm{C}$ por 2 horas. Esta prueba es positiva para C. albicans, quien produce un tubo fino y continuo, sin constricción en el lugar de origen. Posteriormente se realizó la siembra en agar harina de maíz. Una vez que se desarrollan las colonias de levaduras en los medios previamente mencionados, se sembró la levadura en una placa de agar harina de maíz (Corn Meal Agar); sobre el área de inoculación se colocó una lámina cubre objetos, y se dejó en incubación a temperatura ambiente durante 2 días. Al cabo de ese tiempo, se procedió a la observación microscópica de las estructuras características de cada una de las especies de cándida.

A todas las pacientes se les indicó tratamiento médico según las pautas del CDC. Una vez obtenidos los resultados del cultivo, se establecieron grupos según las especies, con el fin de relacionar las mismas con las características clínicas descritas. Los contrastes de las variables nominales de acuerdo a cultivo positivo o negativo para Cándida de basaron en la prueba chi-cuadrado de Pearson, en el caso de las variables continuas, de aplicó la prueba no paramétrica U de Mann-Whitney. Se consideró un valor de contraste significativo si $\mathrm{p}<0,05$. Los datos fueron analizados con JMP-SAS 20.0

\section{RESULTADOS}

Entre las 203 mujeres a las cuales se les realizó cultivo para identificación de la especie de cándida en el flujo vaginal, 165 resultaron positivas para una frecuencia de $81,3 \%(p=0,264)$.

La tabla 1 muestra las características generales de la población. Se encontró que la edad media fue de 33,62 $\pm 12,56$ años. Los oficios del hogar fue la ocupación con mayor frecuencia con un 47,3 \% (96 pacientes). De las 120 pacientes que refirieron coito menos de una vez por semana, 96 de ellas $(80 \%)$ tuvieron 


\section{VULVOVAGINITIS CANDIDIÁSICA: MANIFESTACIONES CLÍNICAS SEGÚN LA ESPECIE DE CÁNDIDA}

Tabla 1. Características generales de la población

\begin{tabular}{lcc}
\hline & Estadístico & $\mathrm{p}$ \\
\hline $\begin{array}{l}\text { Edad (media } \pm \text { DE) } \\
\text { Ocupación n (\%) }\end{array}$ & $33,62 \pm 12,56$ & 0,264 \\
$\quad$ & & \\
$\quad$ Oficios del hogar & $96(47,3)$ & 0,992 \\
$\begin{array}{l}\text { Frecuencia del coito n (\%) } \\
\quad<1 \text { vez/semana }\end{array}$ & $120(59,1)$ & 0,139 \\
$\quad \geq 1$ vez/semana & $83(40,9)$ & 0,033 \\
Método anticonceptivo n $(\%)$ & $61(30,0)$ & 0,171 \\
\hline
\end{tabular}

cultivo positivo $(\mathrm{p}=0,139)$. Entre las 83 pacientes que refirieron coito una vez o más por semana, 69 de ellas $(83,1 \%)$ presentaron cultivo positivo $(p=0,033)$. El uso de método anticonceptivo en general no fue estadísticamente significativo para la aparición de cultivo positivo para cándida. Entre las 61 pacientes (30,0\%) que usaban método anticonceptivo, en la tabla 2 se puede apreciar que la asociación entre diagnóstico micológico positivo para cándida y el uso de ACO fue de $59,2 \%(p=0,002)$.

Tabla 2. Uso de método anticonceptivo en pacientes con cultivo positivo para cándida

\begin{tabular}{lcc}
\hline Método & $\mathrm{n}(\%)$ & $\mathrm{p}$ \\
\hline Anticonceptivos orales & $29(59,2)$ & 0,002 \\
Dispositivos intrauterinos & $6(12,2)$ & 0,054 \\
Barrera & $5(10,2)$ & 0,125 \\
Inyectables & $4(8,2)$ & 0,154 \\
Otros & $5(10,2)$ & 0,474 \\
\hline
\end{tabular}

De las 165 pacientes con resultado positivo, las dos especies con mayor frecuencia fueron Candida albicans con 105 pacientes $(63,6 \%)$ y Candida glabrata con 49 pacientes para un $29,7 \%$. Con menor frecuencia se encontraron Candida parapsilosis, Candida tropicalis y Candida krusei (tabla 3).
Tabla 3. Frecuencia relativa de cándida según especie identificada

\begin{tabular}{lcc}
\hline & $\mathrm{n}=165$ & $\%$ \\
\hline Candida albicans & 105 & 63,6 \\
Candida glabrata & 49 & 29,7 \\
Candida parapsilosis & 6 & 3,6 \\
Candida tropicalis & 4 & 2,4 \\
Candida krusei & 1 & 0,6 \\
\hline
\end{tabular}

En cuanto a las manifestaciones clínicas en las pacientes a las que se realizó cultivo, en la tabla 4 se observa que el eritema vaginal se presentó en 104 pacientes con cultivo positivo y en 9 de las pacientes con cultivo negativo $(\mathrm{p}=0,000)$; con respecto a las excoriaciones, 25 pacientes con cultivo positivo la presentaron y el signo no se presentó en ninguna de las pacientes con cultivo negativo $(p=0,005)$; el flujo vaginal anormal estuvo presente en 164 pacientes con cultivo positivo y en todas las que tuvieron cultivo negativo $(p=0,020)$. El prurito estuvo presente en 136 de las pacientes positivas y en 25 de las negativas $(p=0,022)$; otro signo presente fue el eritema perineal en 33 de las pacientes con microorganismo aislado y en 2 de las mismas con microorganismo ausente $(\mathrm{p}=0,030)$ y por último el eritema vulvar que se presentó en 90 de las pacientes positivas y en 14 de las negativas $(\mathrm{p}=0,049)$. Las demás manifestaciones clínicas evaluadas no tuvieron significancia estadística.

Con respecto a las características del flujo vaginal en las pacientes con cultivo positivo para cándida se encontró que el flujo blanco grumoso fue el único tipo con asociación significativa, estando presente en 154 pacientes con cultivo positivo $(93,9 \%)$ para una $\mathrm{p}=0,048($ tabla 5$)$.

La tabla 6 resume la distribución comparativa de las manifestaciones clínicas en pacientes con cultivo positivo para cándida según la especie identificada. De 
A BAHSAS ZAMORA ET AL.

Tabla 4. Distribución comparativa según las manifestaciones clínicas y cultivo de cándida

\begin{tabular}{lcccccc}
\hline \multirow{2}{*}{ Clínica } & \multicolumn{2}{c}{ Cultivo positivo para cándida } & \multicolumn{2}{c}{ Cultivo negativo para cándida } & Total & $p$ \\
\cline { 2 - 5 } & $\mathrm{n}$ & $\%$ & $\mathrm{n}$ & $\%$ & $\mathrm{n}$ & $p$ \\
\hline Prurito & 136 & 82,4 & 25 & 65,8 & 161 & 0,022 \\
Dolor vaginal & 21 & 12,7 & 4 & 10,5 & 25 & 1,000 \\
Dispareunia & 40 & 24,2 & 5 & 13,2 & 45 & 0,138 \\
Disuria & 49 & 29,7 & 7 & 18,4 & 56 & 0,161 \\
Flujo anormal & 164 & 99,4 & 38 & 100,0 & 202 & 0,020 \\
Edema vulvar & 20 & 12,1 & 3 & 7,9 & 23 & 0,580 \\
Eritema vulvar & 90 & 54,6 & 14 & 36,8 & 104 & 0,049 \\
Eritema perineal & 33 & 20,0 & 2 & 5,3 & 35 & 0,030 \\
Eritema vaginal & 104 & 63,0 & 9 & 23,7 & 113 & 0,000 \\
Excoriaciones & 25 & 15,2 & 0 & 0 & 25 & 0,005 \\
Fisuras & 2 & 1,2 & 0 & 0 & 2 & 1,000 \\
\hline
\end{tabular}

Tabla 5. Distribución comparativa según las características del flujo vaginal en pacientes con cultivo positivo

\begin{tabular}{lccc}
\hline & $\mathrm{n}=164$ & $\%$ & $p$ \\
\hline Blanco grumoso & 154 & 93,90 & 0,048 \\
Blanco fluido & 6 & 3,66 & 0,105 \\
Blanco amarillento & 2 & 1,22 & 0,325 \\
Amarillo & 1 & 0,61 & 0,144 \\
Blanco grisáceo & 1 & 0,61 & 0,235 \\
\hline
\end{tabular}

estas manifestaciones la única que presentó diferencias estadísticamente significativa fue la presencia de excoriaciones en 3 de 4 pacientes $(75 \%)$ con cultivo positivo para Candida tropicalis $(\mathrm{p}=0,012)$.

Al analizar las características del flujo vaginal según la especie de cándida identificada, se encontró que el flujo blanco grumoso fue el más frecuente, presentándose en el $100 \%$ de las infecciones por Candida tropicalis y

Tabla 6. Distribución comparativa según manifestaciones clínicas

en pacientes con cultivo positivo para cándida según la especie

\begin{tabular}{lcccccc}
\hline & \multicolumn{2}{c}{ Especie de cándida identificada } & & \\
& Candida & Candida & Candida & Candida & Candida & $p$ \\
\hline albicans, $\mathrm{n}(\%)$ & glabrata, $\mathrm{n}(\%)$ & krusei, $\mathrm{n}(\%)$ & parapsilosis, $\mathrm{n}(\%)$ & tropicalis, $\mathrm{n}(\%)$ & \\
\hline Dolor vaginal & $83(79,1)$ & $43(87,8)$ & $1(100)$ & $5(83,3)$ & $4(100)$ & 0,582 \\
Dispareunia & $13(12,4)$ & $6(12,2)$ & 0 & $1(16,7)$ & $1(25)$ & 0,710 \\
Disuria & $25(23,8)$ & $14(28,6)$ & 0 & 0 & $1(25)$ & 0,600 \\
Flujo vaginal & $30(28,6)$ & $17(34,7)$ & 0 & $1(16,7)$ & $1(25)$ & 0,808 \\
Edema vulvar & $104(99,1)$ & $49(100)$ & $1(100)$ & $6(100)$ & $4(100)$ & 0,910 \\
Eritema vulvar & $9(8,6)$ & $10(20,4)$ & 0 & $1(16,7)$ & 0 & 0,267 \\
Eritema perineal & $20(53,3)$ & $27(55,1)$ & 0 & $4(66,7)$ & $3(75)$ & 0,681 \\
Eritema vaginal & $60(57,1)$ & $10(20,4)$ & 0 & $1(16,7)$ & $2(50)$ & 0,626 \\
Excoriaciones & $12(11,4)$ & $9(18,4)$ & 0 & $4(66,7)$ & $3(75)$ & 0,317 \\
Fisuras & $2(1,9)$ & 0 & 0 & $1(16,7)$ & $3(75)$ & 0,012 \\
\hline
\end{tabular}




\section{VULVOVAGINITIS CANDIDIÁSICA: MANIFESTACIONES CLÍNICAS SEGÚN LA ESPECIE DE CÁNDIDA}

Tabla 7. Distribución comparativa según características del flujo vaginal en pacientes con cultivo positivo para cándida de acuerdo a la especie

\begin{tabular}{lcccccc}
\hline & \multicolumn{2}{c}{ Especie de cándida identificada } \\
& $\begin{array}{c}\text { Candida } \\
\text { albicans } \\
\mathrm{n}(\%)\end{array}$ & $\begin{array}{c}\text { Candida } \\
\text { glabrata } \\
\mathrm{n}(\%)\end{array}$ & $\begin{array}{c}\text { Candida } \\
\text { krusei } \\
\mathrm{n}(\%)\end{array}$ & $\begin{array}{c}\text { Candida } \\
\text { parapsilosis } \\
\mathrm{n}(\%)\end{array}$ & $\begin{array}{c}\text { Candida } \\
\text { tropicalis } \\
\mathrm{n}(\%)\end{array}$ & $p$ \\
\hline Blanco grumoso & $98(94,2)$ & $47(95,9)$ & $1(100)$ & $4(66,7)$ & $4(100)$ & 0,948 \\
Blanco fluido & $2(1,9)$ & $2(4,1)$ & 0 & $2(33,3)$ & 0 & 0,004 \\
Blanco amarillento & $2(1,9)$ & 0 & 0 & 0 & 0 & 0,885 \\
Amarillo & $1(1,0)$ & 0 & 0 & 0 & 0 & 0,965 \\
Blanco grisáceo & $1(1,0)$ & 0 & 0 & 0 & 0 & 0,965 \\
\hline
\end{tabular}

Candida krusei, en el 95,9\% de los casos de Candida glabrata y 94,2 \% de Candida albicans; aunque tanto para este tipo de flujo como para el blancoamarillento, amarillo y blanco grisáceo no se demostró diferencia estadísticamente significativa. El único flujo que se tradujo en una diferencia estadísticamente significativa fue el blanco fluido, presente en el $33,3 \%$ de las infecciones por Candida parapsilosis $(\mathrm{p}=0,004)($ tabla 7$)$.

\section{DISCUSIÓN}

La presencia de infecciones del tracto genital inferior continúa siendo una de las primeras quejas por las que las pacientes acuden a la consulta ginecológica. En particular las infecciones vaginales por cándida representan una de las patologías a las que frecuentemente se enfrenta el médico, el cual, generalmente, fundamenta tanto el diagnóstico como el tratamiento en las manifestaciones clínicas identificadas. Las cifras de presentación de este tipo de afección son muy variables, dependiendo de los criterios utilizados para establecer el diagnóstico (1, $3)$.

En la presente investigación se logró identificar cándida en pacientes con criterios clínicos diagnósticos de vulvovaginitis candidiásica en $81,3 \%$ de pacientes, similar a lo reportado por Parazzini y cols. (9) quienes reportaron 79,3\% entre 4228 mujeres con sintomatología de infección del tracto genital inferior sugestiva de cándida en edades comprendidas entre 18 y 70 años. Por su parte, Nyirjesy y cols. (16) reportaron solo $24,67 \%$ entre pacientes con síntomas de vulvovaginitis crónica, demostrando que esta sintomatología puede deberse a alteraciones no infecciosas e infecciosas diferentes a la candidiasis.

Con respecto a la presentación según la especie de Cándida, la distribución coincide con reportes nacionales e internacionales, obteniendo casi $64 \%$ de frecuencia de Candida albicans y casi $30 \%$ de Candida glabrata. De La Parte y cols. (1), en Venezuela, encontró Candida albicans en $84 \%$, pero, con respecto a las Candida no albicans encontró resultados diferentes: Candida tropicalis $8 \%$; Candida guilliermondii $5 \%$ y Candida glabrata $3 \%$. Recientemente, se ha documentado un incremento de la proporción de Candida no albicans debido a múltiples factores como el uso indiscriminado de antibióticos, uso de productos de higiene íntima, uso de métodos de anticonceptivos de barrera, etc, que varían de acuerdo a la distribución poblacional (33). 
Las manifestaciones clínicas frecuentemente relacionadas con la vulvovaginitis candidiásica fueron encontradas tanto en pacientes con cultivo positivo como en pacientes con cultivo negativo, esto es debido a que todas las pacientes incluidas en la investigación tenían criterio clínico de sospecha para vulvovaginitis candidiásica. Adicionalmente, se observó que en las pacientes con cultivo positivo se presentó flujo anormal, prurito, eritema vaginal, eritema vulvar, eritema perineal y excoriaciones, todas ellas con diferencia estadísticamente significativa al compararlas con pacientes en las que el cultivo resulto negativo $(\mathrm{p}<0,05)$. Esto se deriva de la patogenicidad de una serie de factores de la cándida tales como: enzimas proteinasas, fosfolipasas, otras enzimas hidrolíticas y mediadores de inflamación liberados por el huésped que explicarían la presentación de los síntomas anteriormente descritos (32 - 34).

El flujo vaginal observado en la VVC se deriva de la reacción del huésped al hongo. Para que la cándida pueda penetrar el epitelio vaginal es necesario que desarrolle hifas y micelios que son capaces de destruir las proteínas vaginales con la consecuente invasión epitelial que trae como consecuencia la liberación de prostaglandinas y bradiquininas capaces de desarrollar cambios inflamatorios, edema, eritema e incremento del flujo vaginal con una mezcla de células epiteliales exfoliadas y leucocitos polimorfonucleares (5).

El prurito se ha explicado como consecuencia de la respuesta inmune Th2 de la vagina ante el hongo, que se caracteriza por la liberación de citoquinas (interleukinas 4; 5 y 10 e inmunoglobulina E) que se unen a los mastocitos liberando histamina y prostaglandina E2 (Pg E2). La histamina induce cambios inflamatorios en la mucosa vulvovaginal, siendo responsable en gran parte del cuadro clínico de la VVC. Por su parte, la Pg E2, en conjunto con las interleukinas, facilitan la transformación de blastosporas a formas germinativas, reacciones que también pueden verse en respuesta a espermicidas y líquido seminal (35).
Todas estas características descritas anteriormente son las que probablemente sean de utilidad para orientar el diagnóstico de vulvovaginitis candidiásica en pacientes que acuden a la consulta ginecológica y los mismos coinciden con los reportados por otros autores $(14,15)$.

Al tratar de correlacionar las manifestaciones clínicas que presentan las pacientes con cultivo positivo para cándida dependiendo de la especie identificada, la característica evaluada que presentó diferencia estadísticamente significativa a favor de la especie Candida tropicalis $(75 \%)$ fue la presencia de excoriaciones con una $\mathrm{p}=0,012$. Kothavade y cols. (36) han estudiado la patogenicidad de la Candida tropicalis, en la que se encuentran involucrados tanto factores dependientes del germen (capacidad de adhesión, enzimas hidrolíticas como proteinasas y lipasas, formación de biopelículas, etc), como factores del huésped (barrera epitelial, inmunidad celular, inmunidad humoral, etc) y concluyen que la interacción de estos factores parece estar involucrada en la presentación de signos y síntomas en la paciente y hasta en la respuesta terapéutica frente a antimicóticos como el fluconazol.

Con relación a las características del flujo vaginal en pacientes con cultivo positivo según la especie, la única característica con diferencia estadísticamente significativa correspondió al flujo blanco fluido, a favor de Candida parapsilosis $333,3 \%$ con una $\mathrm{p}=$ 0,004). Esto puede ser explicado sobre la base del incremento de secreción de aspartil-proteinasa por parte de la $C$. parapsilosis en comparación a las otras especies. Esta proteinasa puede comprometer de manera importante la integridad de la vagina, hidrolizando la inmunoglobulina A secretora, con lo que incrementaría la virulencia de este patógeno (34).

De todo lo anterior se puede concluir que la frecuencia de infecciones por cándida fue de $81,3 \%$, con una prevalencia según la especie, en orden decreciente, 


\section{VULVOVAGINITIS CANDIDIÁSICA: MANIFESTACIONES CLÍNICAS SEGÚN LA ESPECIE DE CÁNDIDA}

de Candida albicans 63,6 \%; Candida glabrata 29,7\%; Candida parapsilosis 3,6\%; Candida tropicalis 2,4\% y Candida krusei $0,6 \%$. Las manifestaciones clínicas encontradas con mayor frecuencia en pacientes con cultivo positivo para cándida fueron: flujo anormal, prurito, eritema vaginal, eritema vulvar, eritema perineal y excoriaciones. Las excoriaciones presentaron asociación significativa con la especie de Candida tropicalis. El flujo blanco fluido presentó asociación significativa con Candida glabrata.

En vista a lo anteriormente expuesto se recomienda no fundamentar el diagnóstico micológico por especies en las manifestaciones clínicas. En pacientes con infecciones recurrentes se debería documentar el diagnóstico micológico.

\section{REFERENCIAS}

1. De La Parte M, Mendoza M, Brito A. Identificación de especies de levaduras del género Candida provenientes de pacientes con vulvovaginitis. Vitae AC Biom Dig [Internet]. 2006 [consultado 14 de mayo de 2011]; 27 [aprox. 6 páginas]. Disponible en: https://vitae.ucv.ve/ pdfs/VITAE_87.pdf

2. Nyirjesy P, Sobel JD. Vulvovaginal candidiasis. Obstet Gynecol Clin North Am. 2003; 30(4):671-684. doi: 10.1016/s0889-8545(03)00083-4. PMID: 14719844.

3. Pappas PG, Bergamo B. Superficial and mucosal fungal infections. En: Maertens JA, Marr KA, editores. Diagnosis of fungal infections. London: Informa Healthcare; 2007. p. $153-169$.

4. Ahmad I, Owais M, Shahid M, Aqil F. Combating fungal infections. Problems and remedy. Berlín: Springer; 2010.

5. Figueroa J. Candidiasis vulvovaginal. En: Casanova G, Ortiz F, Reyna F, editores. Infecciones de transmisión sexual. México: Editorial Alfil, 2004. p. 267-277.

6. Faro S. Vaginitis. Differential Diagnosis and Management. Lancashire (UK): The Parthenon Publishing Group; 2005.

7. Martins HP, da Silva MC, Paiva LC, Svidzinski TI, Consolaro ME. Efficacy of fluconazole and nystatin in the treatment of vaginal Candida species. Acta Derm Venereol. 2012; 92(1):78-82. doi: 10.2340/000155551194. PMID: 21918792.

8. Handa VL, Stice CW. Fungal culture findings in cyclic vulvitis. Obstet Gynecol. 2000; 96(2):301-303. doi: 10.1016/s0029-7844(00)00898-x. PMID: 10908782.

9. Parazzini F, Di Cintio E, Chiantera V, Guaschino S. Determinants of different Candida species infections of the genital tract in women. Sporachrom Study Geoup. Eur J Obstet Gynecol Reprod Biol. 2000; 93(2):141145. doi: 10.1016/s0301-2115(00)00289-x. PMID: 11074134.

10. Bauters TG, Dhont MA, Temmerman MI, Nelis HJ. Prevalence of vulvovaginal candidiasis and susceptibility to fluconazole in women. Am J Obstet Gynecol. 2002; 187(3):569-574. doi: 10.1067/ mob.2002.125897. PMID: 12237629.

11. Moragues MD, Omaetxebarria MJ, Elguezabal N, Bikandi J, Quindós G, Coleman DC, et al. Serological differentiation of experimentally induced Candida dubliniensis and Candida albicans infections. J Clin Microbiol. 2001; 39(8):2999-3001. doi: 10.1128/ JCM.39.8.2999-3001.2001. PMID: 11474033; PMCID: PMC88280.

12. McGinnis MR, Tilton RC. Yeasts. En: Howard BJ, Keiser JF, Smith TF, Weissfeld M, Tilton RC, editores. Clinical and pathoge $\neg$ nic microbiology. 2nd ed. St Louis: Mosby, 1994. p. 615-24.

13. Cassone A, De Bernardis F, Santoni G. Anticandidal immunity and vaginitis: novel opportunities for immune intervention. Infect Immun. 2007; 75(10):4675-4686. doi: 10.1128/IAI.00083-07. Epub 2007 Jun 11. PMID: 17562759; PMCID: PMC2044548.

14. Grigoriou O, Baka S, Makrakis E, Hassiakos D, Kapparos G, Kouskouni E. Prevalence of clinical vaginal candidiasis in a university hospital and possible risk factors. Eur J Obstet Gynecol Reprod Biol. 2006; 126(1):121-125. doi: 10.1016/j.ejogrb.2005.09.015. Epub 2005 Oct 26. PMID: 16256258.

15. Richter SS, Galask RP, Messer SA, Hollis RJ, Diekema DJ, Pfaller MA. Antifungal susceptibilities of Candida species causing vulvovaginitis and epidemiology of recurrent cases. J Clin Microbiol. 2005; 43(5):21552162. doi: 10.1128/JCM.43.5.2155-2162.2005. PMID: 15872235; PMCID: PMC1153777.

16. Nyirjesy P, Seeney SM, Grody MH, Jordan CA, Buckley HR. Chronic fungal vaginitis: the value of cultures. Am J Obstet Gynecol. 1995; 173(3 Pt 1):820-823. doi: 10.1016/0002-9378(95)90347-x. PMID: 7573250.

17. Lopes Consolaro ME, Aline Albertoni T, Shizue Yoshida C, Mazucheli J, Peralta RM, Estivalet Svidzinski TI. Correlation of Candida species and symptoms among patients with vulvovaginal candidiasis in Maringá, Paraná, Brazil. Rev Iberoam Micol [Internet]. 2004 [consultado 27 de octubre de 2013]; 21(4):202-205. PMID: 15709802. Disponible en: https://pubmed.ncbi. 
nlm.nih.gov/15709802/

18. Zeng J, Zong LL, Mao T, Huang YX, Xu ZM. Distribution of Candida albican genotype and Candida species is associated with the severity of vulvovagianl candidiasis. Nan Fang Yi Ke Da Xue Xue Bao [Internet]. 2011 [consultado 27 de octubre de 2013]; 31(10):1649-1653. PMID: 22027762. Disponible en: https://pubmed.ncbi.nlm.nih.gov/22027762/

19. Achkar JM, Fries BC. Candida infections of the genitourinary tract. Clin Microbiol Rev. 2010; 23(2):253-273. doi: 10.1128/CMR.00076-09. PMID: 20375352; PMCID: PMC2863365.

20. Sobel JD, Faro S, Force RW, Foxman B, Ledger WJ, Nyirjesy PR, et al. Vulvovaginal candidiasis: epidemiologic, diagnostic, and therapeutic considerations. Am J Obstet Gynecol. 1998; 178(2):203-11. doi: 10.1016/s0002-9378(98)80001-x. PMID: 9500475.

21. Sobel JD. Vulvovaginal candidosis. Lancet. 2007; 369(9577):1961-71. doi: 10.1016/S01406736(07)60917-9. PMID: 17560449.

22. Spinillo A, Capuzzo E, Gulminetti R, Marone P, Colonna L, Piazzi G. Prevalence of and risk factors for fungal vaginitis caused by non-albicans species. Am J Obstet Gynecol. 1997; 176(1 Pt 1):138-41. doi: 10.1016/s0002-9378(97)80026-9. PMID: 9024104.

23. Iglesias J, Saldivar D, Tijerina R, González G, Garza G, Rosales T. Especies de Cándida no albicans en la consulta de ginecología. Med Univer [Internet]. 2007 [consultado 14 de mayo de 2011]; 9(37): 161-165. Disponible en: https://www.medigraphic.com/pdfs/ meduni/mu-2007/mu074c.pdf

24. Trama JP, Adelson ME, Raphaelli I, Stemmer SM, Mordechai E. Detection of Candida species in vaginal samples in a clinical laboratory setting. Infect Dis Obstet Gynecol. 2005; 13(2):63-67. doi: 10.1080/10647440400025629. PMID: 16011995; PMCID: PMC1784561.

25. Vermitsky JP, Self MJ, Chadwick SG, Trama JP, Adelson ME, Mordechai E, et al. Survey of vaginalflora Candida species isolates from women of different age groups by use of species-specific PCR detection. J Clin Microbiol. 2008; 46(4):1501-1503. doi: 10.1128/ JCM.02485-07. Epub 2008 Feb 27. PMID: 18305136; PMCID: PMC2292962.

26. Fuenmayor-Boscán A, Paz-Montes A, FuenmayorBoscánA,AcostaN.Diagnósticoclínico presuntivo versus diagnóstico microbiológico en mujeres con leucorrea.
Rev Soc Ven Microbiol [Internet]. 2009 [consultado 27 de octubre de 2013]; 29(1): 26-33. Disponible en: $\quad$ http://ve.scielo.org/scielo.php?script=sci arttext\&pid=S1315-25562009000100006

27. Torres K, Soto A, Sandrea D, Villalobos M, Rodríguez A, Hassanhi M, et al. Candidiasis vaginal en primigestas. Rev Obstet Ginecol Venez [Internet]. 2005 [consultado 27 de octubre de 2013]; 65(2):55-58. Disponible en: $\quad$ http://ve.scielo.org/scielo.php?script=sci arttext\&pid=S0048-77322005000200002

28. Odds FC. Genital candidosis. Clin Exp Dermatol. 1982; 7(4):345-54. doi: 10.1111/j.1365-2230.1982. tb02441.x. PMID: 7127883.

29. Yeo SF, Wong B. Current status of nonculture methods for diagnosis of invasive fungal infections. Clin Microbiol Rev. 2002; 15(3):465-484. doi: 10.1128/ cmr.15.3.465-484.2002. PMID: 12097252; PMCID: PMC118074.

30. Workowski KA, Bolan GA; Centers for Disease Control and Prevention. Sexually transmitted diseases treatment guidelines, 2015. MMWR Recomm Rep [Internet]. 2015 [consultado 18 de noviembre de 2015]; 64(RR03):1-137. PMID: 26042815; PMCID: PMC5885289. Disponible en: https://www.cdc.gov/mmwr/preview/ mmwrhtml/rr6403a1.htm

31. Achkar JM, Fries BC. Candida infections of the genitourinary tract. Clin Microbiol Rev. 2010; 23(2):253-273. doi: 10.1128/CMR.00076-09. PMID: 20375352; PMCID: PMC2863365.

32. Fidel PL Jr. Immunity in vaginal candidiasis. Curr Opin Infect Dis. 2005; 18(2):107-111. doi: 10.1097/01. qco.0000160897.74492.a3. PMID: 15735412.

33. Arenas R. Micología médica ilustrada. 3ra Ed. México: McGraw Hill Interamericana Editores; 2008.

34. Trofa D, Gácser A, Nosanchuk JD. Candida parapsilosis, an emerging fungal pathogen. Clin Microbiol Rev. 2008; 21(4):606-625. doi: 10.1128/CMR.00013-08. PMID: 18854483; PMCID: PMC2570155.

35. Farinati AE, Mormandi JO, Tilli M. Infecciones en Ginecología y Obstetricia. Del Diagnóstico al Tratamiento. Buenos Aires: Color Press SRL. 1988.

36. Kothavade RJ, Kura MM, Valand AG, Panthaki MH. Candida tropicalis: its prevalence, pathogenicity and increasing resistance to fluconazole. J Med Microbiol. 2010; 59(Pt 8):873-880. doi: 10.1099/jmm.0.013227-0. Epub 2010 Apr 22. PMID: 20413622

Recibido 20 de julio de 2020 Aprobado 18 de septiembre de 2020 